\title{
Third-order polarizability of interlayer excitons in hetero-bilayers
}

\author{
M. F. C. Martins Quintela ${ }^{1,2}$, J. C. G. Henriques ${ }^{1,2}$, N. M. R. Peres ${ }^{1,2}$ \\ ${ }^{1}$ Department and Centre of Physics, University of Minho, \\ Campus of Gualtar, 4710-057, Braga, Portugal and \\ ${ }^{2}$ International Iberian Nanotechnology Laboratory (INL), \\ Av. Mestre José Veiga, 4715-330, Braga, Portugal
}

\begin{abstract}
In this paper, we employ a fully microscopic approach to the study of interlayer excitons in hetero-bilayers. We use Fowler's and Karplus' method to access the dynamical polarizability of non-interacting interlayer excitons in a $\mathrm{WSe}_{2} / \mathrm{WS}_{2}$-based van der Waals heterostructure. Following from the calculation of the linear polarizability, we consider Svendsen's variational method to the calculation of the dynamic third-order polarizability. With this variational method, we study both two-photon absorption and third-harmonic generation processes for interlayer excitons in a $\mathrm{WSe}_{2} / \mathrm{WS}_{2}$ hetero-bilayer, discussing the various selection rules of intra-excitonic energy level transitions.
\end{abstract}

\section{INTRODUCTION}

The advent of the study of two-dimensional layers with atomic thickness has renewed the interest in transition-metal dichalcogenides (TMDs) and their optical/optoelectronic properties [1. These materials, which have been studied in their bulk form since the 1960's [2, 3], have been shown to be good candidates for various optical and optoelectronic applications [4-7].

The optical response of these materials is dominated by excitons [8], and optically bright exciton absorption peaks have been shown to correspond to the excitation of states in the $n s$ series [9, 10]. As for $n p$-series excitons, these can be controlled magnetically despite being optically dark in TMDs [11. The selection rules of excitons in TMDs for absorption experiments have been recently thoroughly studied 12 .

The linear dielectric response of TMDs consists of two distinct regimes: the interband regime, where electrons from the valence band are excited to the conduction band, leaving being a hole [6, 13]. The attractive electrostatic interaction between this newly-formed electronhole pair leads to the formation of a bound state (exciton), and its $n s$ states are optically active and can be observed in absorption measurements [14]. The second regime, characterized by intra-exciton transitions, consists on the transition between the excitonic ground state $(1 s)$ and the empty $n p$ states [15, 16]. Each of the $1 s \rightarrow n p$ transitions [17-19] is characterized by a peak in the dynamical polarizability which, in turn, determines the dielectric response of the system in a pump-probe experiment [20, 21].

Interlayer excitons are formed when two monolayers are brought together with type-II band alignment, where the conduction band minimum and the valence band maximum are located in different layers [22, 23]. This allows the intralayer electron-hole pairs to tunnel into interlayer excitons (Fig. 1-left), which have a significantly longer life-time due to the small overlap of the individual electron and hole wave functions [24, 25]. Additionally, interlayer excitons exhibit luminescence at lower energies than their intralayer counterpart [26] which, together
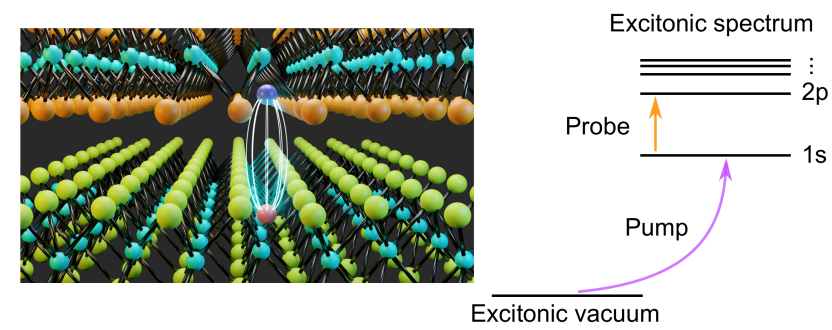

Figure 1. Left: Interlayer exciton in a van der Waals heterostructure. Right: Diagram of pump-probe experiments for observing $n p$ (dark) excitonic states.

with their lower binding energies, allows for an easier identification of the specific species in question in polarizability measurements 21. Recently, Merkl et al. [21. were able to observe the transition between interlayer and intralayer excitonic phases in van der Waals heterostructures. This observation was performed via the measurement of the linear dielectric function of the excitons in a pump-probe experiment (Fig. 1-right), observing a significant shift in maximum of the optical conductivity.

The excitonic energy levels are known to depend on whether the system is confined or not (for example, the Rydberg series for the two-dimensional Hydrogen atom is strongly affected by radial confinement [27]). Following recent advancements in the fabrication of TMD-based meta-materials with atomic precision [28, 29], we focus our attention on radially confined interlayer excitons in a $\mathrm{WSe}_{2} / \mathrm{WS}_{2}$ bilayer.

From studying the dynamic polarizability of the excitonic states, the full dielectric response of the excited sample can be extracted. This transition from polarizability to dielectric response is one from the microscopic (individual exciton) to the macroscopic (when many excitons form a low-density exciton gas). The susceptibility of this exciton gas would be given, in a simplified form, as $\chi_{\mathrm{ex}}^{\text {inter }}=\frac{N_{X} \alpha}{\epsilon_{0}}$, with $N_{X}$ the exciton density in the twodimensional material, $\alpha$ the linear polarizability, and $\epsilon_{0}$ the vacuum dielectric constant. Separating the real and 
imaginary part of the susceptibility, these can be experimentally measured [20, 21, 30, 31] and, therefore, would provide a good comparison point for the results we obtain for the linear polarizability.

The non-linear polarizabilities of the hydrogen atom have been thoroughly studied throughout the years [32, 33. providing a good starting point for the study of these same non-linear processes in excitons. Recently, the non-linear optical effects from intra-excitonic transitions have been the focus of many theoretical and experimental works. This has been performed for several TMDs, including but not limited to $\mathrm{WSe}_{2}\left[34,35\right.$, and $\mathrm{WS}_{2}$ [36, 37, as well as hBN homostructures [38]. Through doping processes, the optical non-linear properties of TMDs can be tuned [39] which, along with their large non-linear optical coefficients [12, increases their feasibility for practical aplications [40] (e.g., as optical modulators).

We begin this paper by discussing the necessary modifications to the Rytova-Keldysh potential [41, 42] when dealing with interlayer excitons, followed by analyzing an harmonic oscillator approximation to this potential which will motivate the chosen variational wave-function. After defining this ansatz, we outline the complete set of basis functions we will use to approximate the excited states of the exciton. The necessary material-dependent set of parameters characteristic of the specific van der Waals heterostructure in question will also be discussed, obtaining the desired energy levels.

In Section III we outline Fowler's and Karplus' method [43, 44] to compute the dynamical polarizability of noninteracting excitons. We then apply this same method to interlayer excitons in a $\mathrm{WSe}_{2} / \mathrm{WS}_{2}$-based van der Waals heterostructure, comparing the obtained resonances against the transitions calculated both numerically and variationally via a finite-basis approach.

In Section IV] we turn to the non-linear response of the excitons [45, 46]. We focus our discussion on the thirdorder polarizability, first outlining a variational procedure based on the ideas of both Karplus and Svendsen [43, 47, 48] for the calculation of the intra-excitonic third-order polarizability. This procedure was recently studied by Henriques et al., applied to for the study of the two-photon absorption for excitons in $\mathrm{WSe}_{2}$ [49]. After outlining this variational method, we look at the two-photon absorption for excitons in $\mathrm{WSe}_{2} / \mathrm{WS}_{2}$, discussing the various intra-excitonic transitions observed. Finally, we consider the third-harmonic generation, first with an extremely small broadening as to clearly differentiate each individual resonance, and afterwards with two much larger broadenings as to ascertain the feasibility of experimental detection of each peak. A diagrammatic representation of the various transitions is also presented for both the two-photon absorption and third-harmonic generation processes. V]

We finish the paper with our closing remarks in Section

\section{INTERLAYER RYTOVA-KELDYSH POTENTIAL}

We begin this section by discussing the Hamiltonian in which we focus our attention. This will lead to the discussion of the necessary modifications to the RytovaKeldysh potential when dealing with interlayer excitons. We define the basis for our variational approach to the excitonic states (starting by the $n p$-series states for Section III and then the $n s-$ and $n d-$ series for Section IV]. We finish this section by comparing the various variational energies for the excitonic ground state against the result from numerical integration of the Schrödinger equation in a log-grid [50 52] via the Numerov shooting method.

\section{A. Model Hamiltonian in the Dipole Approximation}

Let us now begin by considering the following Hamiltonian (in atomic units, as will be used throughout this paper) in the dipole approximation

$$
\begin{aligned}
H & =H_{0}-\mathbf{r} \cdot \mathbf{F}(t) \\
& =-\frac{1}{2 \mu} \nabla^{2}+V(r)-\mathbf{r} \cdot \mathbf{F}(t),
\end{aligned}
$$

where $\mu$ is the reduced mass of the electron-hole system, $\nabla^{2}$ is the Laplacian operator (taken in polar coordinates throughout this paper), $V(r)$ is a potential energy term and $\mathbf{F}(t)$ is an external time-dependent field. This external field will be initially ignored as to first outline the variational methods and wave functions that will be used, and its action will be studied in Sections III] and IV] when the methods to obtain both the linear and the third-order dynamical polarizability are described, respectively.

Considering this same electron-hole system in a van der Waals (vdW) heterostructure, their potential energy is accurately modeled by the Rytova-Keldysh potential [41, 42]

$$
V_{R K}(r)=-\frac{\pi}{2 r_{0}}\left[\mathbf{H}_{0}\left(\kappa \frac{r}{r_{0}}\right)-\mathbf{Y}_{0}\left(\kappa \frac{r}{r_{0}}\right)\right]
$$

with $\kappa$ the mean dielectric constant of the media, $r_{0}$ an intrinsic parameter of the 2D material (interpretable as an in-plane screening length), and $\mathbf{H}_{0}, \mathbf{Y}_{0}$ the Struve$\mathrm{H}$ and Bessel-Y (second kind) special functions of zeroth order, respectively. The polarizability of intralayer excitons is discussed in detail in [49, 53].

When considering interlayer excitons, a minimum separation originating from the physical distance between the two layers appears in the Rytova-Keldysh potential. The interlayer modified Rytova-Keldysh potential then 
reads 54

$$
\begin{aligned}
V_{\mathrm{i}-\mathrm{RK}}(r)=- & \frac{\pi}{2 r_{0}}\left[\mathbf{H}_{0}\left(\kappa \frac{\sqrt{r^{2}+d^{2}}}{r_{0}}\right)-\right. \\
& \left.-\mathbf{Y}_{0}\left(\kappa \frac{\sqrt{r^{2}+d^{2}}}{r_{0}}\right)\right],
\end{aligned}
$$

with $d$ this interlayer separation distance.

Presenting the same Coulomb tail behaviour as the Rytova-Keldysh potential [Eq. [2p] at large distances, the finite interlayer separation $d$ eliminates the logarithmic divergence at the origin. The absence of this divergence makes it so that an inverse-exponential ansatz for the excitonic wave function [55] is not the most adequate. This, together with the parabolic nature of the interlayer modified Rytova-Keldysh potential near $r=0$, motivates the ansatz that will be obtained in the following section.

\section{B. Gaussian-Based Ansatz}

Ahead we introduce a Gaussian wave function to describe the ground state of the interlayer $1 s$ state analytically. In order to motivate this choice, we show below that the potential Eq. (3) can, indeed, be approximated by a parabolic potential near $r=0$.

In this regime, Eq. (3) can be expanded up to second order in $r$ as

$$
V(r) \approx-V_{0}+\gamma r^{2}
$$

where

$$
\begin{gathered}
V_{0}=\frac{\pi}{2 r_{0}}\left[\mathbf{H}_{0}\left(\kappa \frac{d}{r_{0}}\right)-\mathbf{Y}_{0}\left(\kappa \frac{d}{r_{0}}\right)\right], \\
\gamma=-\frac{\pi \kappa}{4 d r_{0}^{2}}\left[\mathbf{H}_{1}\left(\kappa \frac{d}{r_{0}}\right)-\mathbf{Y}_{-1}\left(\kappa \frac{d}{r_{0}}\right)\right] .
\end{gathered}
$$

Considering this potential, the Hamiltonian will be

$$
H=-\frac{\hbar^{2}}{2 \mu} \nabla^{2}-V_{0}+\gamma r^{2}
$$

For the harmonic oscillator it is well known $[56,[58$ that the $1 s$ function is proportional to a Gaussian, that is, we have

$$
\Psi(\eta) \propto e^{-\eta / 2},
$$

where $\eta=\mu \omega r^{2} / \hbar$ and $\omega=\sqrt{2 \gamma / \mu}$. As such, an ansatz based on this solution is given by

$$
\psi(r)=\mathcal{C} e^{-\frac{r^{2}}{2 \beta}}
$$

where $\mathcal{C}$ is a normalization constant and $\beta$ is a variational parameter. As we are interested in studying interlayer

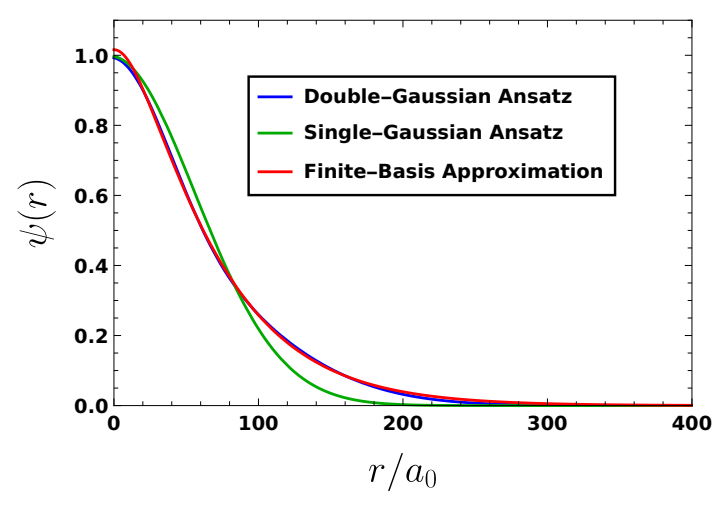

Figure 2. Radial profile of the Double-Gaussian Ansatz [Eq. [8] ], the Gaussian Ansatz [Eq. (7)] and the Finite-Basis Approximation (Sec. IIC) of the ground-state excitonic wave function.

excitons confined to a finite radius, a multiplicative factor is added to impose boundary conditions,

$$
\psi_{0}(r)=\mathcal{C} e^{-\frac{r^{2}}{2 \beta}}(R-r),
$$

where $R$ is the radius of the enclosure, considered $R=$ 1200 throughout this paper (in atomic units). This radius is considered sufficiently large such that the variational wave functions become zero significantly before the boundary is reached (clearly seen in Fig. 2, where the wave functions are already zero at around $\sim R / 3$ ).

Similarly to the ansatz defined by Pedersen in [55], we modify Eq. (7) into a sum of two gaussians, given by

$$
\psi_{0}(r)=\mathcal{C}\left(e^{-\frac{r^{2}}{2 a}}+b e^{-\frac{r^{2}}{2 c}}\right)(R-r),
$$

which will the be ansatz of the excitonic ground state we will consider throughout this article. Additionally, a visual comparison of Eq. (7) against Eq. (8), as well as the approximate wave function obtained via the method described in Sec. IIC is present in Fig. 2 Clearly the ansatz (8) performs much better than the ansatz (7).

\section{Variational Approach from Boundary Conditions}

In certain conditions, namely for a small interlayer separation $d$, the obtained eigenvalues from minimization of the Hamiltonian

$$
H_{0}=-\frac{1}{2 \mu} \nabla^{2}+V_{\mathrm{i}-\mathrm{RK}}(r)
$$

with the ansatz from Eq. (8) are not significantly different from those obtained from numerical integration (a small difference is present for the present case in Table II). Additionally, obtaining higher energy states involves orthogonalization against Eqs. (7) or (8), followed by normalization, a process whose complexity increases substantially when increasing the number of excited states [59]. 
A different approach to obtaining variational solutions to the Schrödinger equation with the interlayer modified Rytova-Keldysh is by integrating the Hamiltonian matrix elements for a complete basis whose elements obey the necessary boundary conditions. Truncating this basis and diagonalizing the Hamiltonian matrix, we obtain a set of variational wave functions that converge towards the real wave functions of the system as the basis size increases.

Following 60, a basis of functions can be constructed from the solutions of the circular infinite well, given by

$$
\psi_{n, l}(r, \varphi)=\frac{C_{n, l}}{\sqrt{2 \pi}} e^{i l \varphi} J_{l}\left(z_{n, l} \frac{r}{R}\right),
$$

where $J_{l}(x)$ is the Bessel function of the first kind of order $l, z_{n, l}$ is the $n$-th zero of $J_{l}(z)$, and $C_{n, l}$ is a normalization constant given by

$$
C_{n, l}=\sqrt{\frac{2}{R^{2} J_{|l|+1}^{2}\left(z_{n, l}\right)}} .
$$

As both $V_{R K}(r)$ and $V_{i-R K}(r)$ are invariant under rotations, the quantum number $l$ is well-defined. As such, we can integrate the Hamiltonian and diagonalize it for a finite number of functions with fixed $l$. Knowing this, we will start the discussion by outlining the necessary material dependent parameters for a $\mathrm{WSe}_{2} / \mathrm{WS}_{2}$ van der Waals heterostructure.

\section{Material Dependent Parameters in $\mathrm{WSe}_{2} / \mathrm{WS}_{2}$}

To perform the necessary calculations and obtain the wave functions and energy eigenvalues, we must substitute the material-specific parameters characteristic of a $\mathrm{WSe}_{2} / \mathrm{WS}_{2}$ van der Waals (vdW) heterostructure [21.

The reduced mass of interlayer excitons in this vdW heterostructure is $\mu=0.15$ and, as such, the effective Bohr radius will be $a_{0} \approx 0.581 \AA$. The thickness of each layer is considered $d_{\text {mat }}=5.7 \AA$ and the gap distance is $d_{\text {gap }}=1 \AA$. As such, the effective interlayer distance will be

$$
d_{\text {eff }}=\frac{1}{2} d_{\text {mat }}+d_{\text {gap }}+\frac{1}{2} d_{\text {mat }}=6.7 \AA .
$$

The permittivities for each material are given by

$$
\epsilon_{(n)}=\sqrt{\epsilon_{(n)}^{\perp} \epsilon_{(n)}^{\|}},
$$

where $\epsilon_{(n)}^{\|}$and $\epsilon_{(n)}^{\perp}$ are the in- and out-of-plane relative permittivities of the material $n$. The numerical values of each component of the relativie permittivities in each material is present in Table I] Knowing the permittivities in each material, the average permittivity of the vdW heterostructure is

$$
\kappa_{\mathrm{mat}}=\frac{\epsilon_{\mathrm{WSe}_{2}}+\epsilon_{\mathrm{WS}_{2}}}{2}=9.31
$$

\begin{tabular}{|c|c|c|}
\hline & $\mathrm{WSe}_{2}$ & $\mathrm{WS}_{2}$ \\
\hline \hline$\epsilon_{(n)}^{\perp}$ & 7.5 & 6.3 \\
\hline$\epsilon_{(n)}^{\|}$ & 13.36 & 11.75 \\
\hline \hline$\epsilon_{(n)}$ & 10.01 & 8.604 \\
\hline
\end{tabular}

Table I. In-plane, out-of-plane, and total relative permittivities of $\mathrm{WSe}_{2}$ and $\mathrm{WS}_{2}$.

\begin{tabular}{|c|c|}
\hline & $1 s$ State \\
\hline \hline Finite Basis & -0.00143378 \\
\hline Shooting Method & -0.00144375 \\
\hline Double-Gaussian [Eq. [8] ] & -0.00142879 \\
\hline Gaussian [Eq. [7] ] & -0.00136314 \\
\hline
\end{tabular}

Table II. Comparison of the different estimates for the excitonic ground-state energy eigenvalue for a $\mathrm{WSe}_{2} / \mathrm{WS}_{2}$ heterostructure. The interlayer distance is given in Eq. (11)

Regarding the screening length $r_{0}$, this parameter can be separated as a sum for each layer (as described in [54])

$$
r_{0} \rightarrow r_{0}^{(1)}+r_{0}^{(2)}
$$

where $r_{0}^{(n)}$ denotes the screening length for the layer $n$. The screening length for each individual layer can be obtained as [20]

$$
r_{0}^{(n)}=\frac{\epsilon_{n}^{2}-1}{2 \epsilon_{n}} d_{n} \sqrt{\frac{\epsilon_{n}^{\|}}{\epsilon_{n}^{\perp}}},
$$

where $d$ is the thickness of the layer. As the in- and outof-plane relative permittivities of the two materials are known, the total screening length is

$$
r_{0}=70.73 \AA \text {. }
$$

Choosing a basis size of 120 and the same enclosure radius as in Sec. II B $(R=1200)$, the ground-state energy eigenvalue is (in both atomic units and $\mathrm{meV}$ )

$$
E_{1,0}=-0.00143378=-39.0152 \mathrm{meV} \text {. }
$$

A comparison against both variational ansatze [Eqs. (7) and (8)] and the numerical results from considering the shooting method in a log-grid is given in Table II]

\section{DYNAMICAL VARIATIONAL METHOD FOR LINEAR POLARIZABILITY}

In this section we will briefly outline Fowler's and Karplus' 43, 44 method to compute the dynamical polarizability of various systems. This discussion will be along the same lines as in 53, serving as a quick outline of the procedure as some results will be necessary further ahead. We apply this method to calculate the linear polarizability of interlayer excitons in a $\mathrm{WSe}_{2} / \mathrm{WS}_{2}$ circular 
dot, comparing the obtained peaks with the calculated $1 s \rightarrow n p$ transitions.

Looking back to Eq. (1), we consider (without loss of generality) the external field pointing along the $-x$ direction. Closely following the approach presented in [53], first outlined by Fowler and Karplus [43, 44], the time-dependent Schrödinger equation reads

$$
\left[H_{0}+x F(t)\right]|\psi(t)\rangle=i \frac{\partial}{\partial t}|\psi(t)\rangle .
$$

where $|\psi(t)\rangle$ describes the wave function of the system in the presence of the electric field $\mathbf{F}$. The dynamical polarizability is defined as

$$
\alpha(\omega)=-\left\langle\psi_{0}|x| \psi_{1}^{+}\right\rangle-\left\langle\psi_{0}|x| \psi_{1}^{-}\right\rangle
$$

with $\psi_{0}$ the Gaussian ansatz of Eq. (8) and $\psi_{1}^{ \pm}$the excited $p$-series states, such that

$$
E(\omega)=E_{0}-\frac{1}{2} \alpha(\omega) F^{2} .
$$

As we are working on a finite disk of radius $R$, Bessel functions of the first kind are an appropriate complete set of functions to describe a problem in such a geometry, as defined in Eq. (10). Using these functions as a basis, we write $\psi_{1}^{ \pm}(\mathbf{r})$ as

$$
\psi_{1}^{ \pm}(\mathbf{r})=\cos \theta \sum_{n=1}^{N} c_{n}^{ \pm} J_{1}\left(\frac{z_{1, n} r}{R}\right),
$$

where $J_{1}(z)$ is the Bessel function of the first kind of order $1, z_{1, n}$ is the $n$-th zero of $J_{1}(z), N$ is the number of Bessel functions we choose to use, and $\left\{c_{n}^{ \pm}\right\}$are a set of coefficients yet to be determined. As proposed in Refs. [43, 61 63, the values of $\left\{c_{n}^{ \pm}\right\}$are determined from the minimization of the functional

$$
\begin{aligned}
\mathcal{J}_{ \pm} & =\int d \mathbf{r} \psi_{1, n}^{ \pm}(\mathbf{r})\left[H_{0}-E_{0} \pm \hbar \omega\right] \psi_{1, n}^{ \pm}(\mathbf{r})+ \\
& +2 \int d \mathbf{r} \psi_{1, n}^{ \pm}(\mathbf{r}) r \cos \theta \psi_{0}(\mathbf{r}),
\end{aligned}
$$

The discussion of the minimization of the $\mathcal{J}$ functional and the computation of the $c_{n}^{ \pm}$coefficients is performed in Appendix A.

The dynamical polarizability is then computed by substituting Eq. 177 into Eq. 15, together with the $c_{n}^{ \pm}$ coefficients discussed in Appendix A, being written as

$$
\begin{aligned}
\alpha(\omega)=- & g_{v} \pi \sum_{n=1}^{N}\left(c_{n}^{+}+c_{n}^{-}\right) \times \\
& \times \int_{0}^{R} J_{1}\left(\frac{z_{1 n} r}{R}\right) r \psi_{0}(r) r d r .
\end{aligned}
$$

where $g_{v}=2$ is the valley degeneracy. The $\omega$ dependence on the right hand side is present in the coefficients $c_{n}^{ \pm}$[more explicitly, this dependence is present in the $\mathbb{M}$

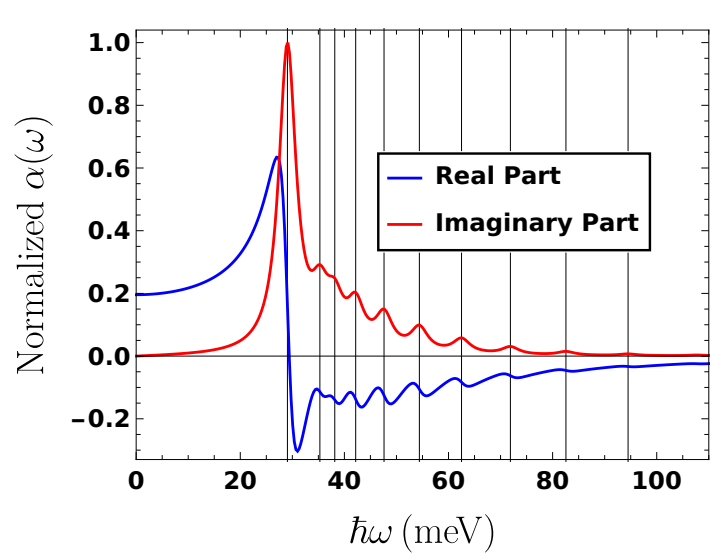

Figure 3. Real (blue) and imaginary (red) parts of the normalized polarizability for interlayer excitons in a $\mathrm{WSe}_{2} / \mathrm{WS}_{2}$ heterostructure. The vertical black lines represent the excitonic $1 s \rightarrow n p$ transitions.

matrix (Eq. A10 from the $g_{i}^{ \pm}(\omega)$ term, as defined in Eq. A99]. We remark that choosing for the ground state wave function a variational function allows us to write Eq. 19p exclusively in terms of analytical functions. This considerably simplifies the numerical minimization.

The polarizability for interlayer excitons in a $\mathrm{WSe}_{2} / \mathrm{WS}_{2}$ heterostructure is plotted in Fig. 3, normalized by its value at the maximum for the excitonic $1 s \rightarrow 2 p$ transition (with $E_{1 s \rightarrow 2 p} \sim 29.0576 \mathrm{meV}$ ) for easier visualization. Additionally, a brief comparison of the linear polarizability for interlayer and intralayer excitons is made in Appendix B.

Having outlined and obtained the linear polarizability in Fig. 3, we now turn our attention to the third-order polarizability of interlayer excitons in this same vdW heterostructure.

\section{SVENDSEN'S METHOD FOR THE THIRD-ORDER POLARIZABILITY}

Having outlined Fowler's and Karplus' method in Section III], we will now consider a similar method for the third-order polarizability, following the procedure delineated by Svendsen [47, 48] and recently applied by Henriques et al [49, to intralayer excitons in TMDs.

Maintaining the definition for the $\mathbf{c}^{ \pm}$coefficients given in Eq. A10, and following closely [43, 48, 49, we write the functional

$$
\begin{aligned}
& \mathcal{K}=\left\langle\xi_{\alpha \beta}\left(\omega_{a}, \omega_{b}\right)\left|H_{0}-E_{0}+\omega_{a}+\omega_{b}\right| \xi_{\alpha \beta}\left(\omega_{a}, \omega_{b}\right)\right\rangle+ \\
& \quad+\left\langle\xi_{\alpha \beta}\left(\omega_{a}, \omega_{b}\right)\left|\mathbf{d}_{\beta}\right| \psi_{\alpha}\left(\omega_{a}\right)\right\rangle+\left\langle\psi_{\alpha}\left(\omega_{a}\right)\left|\mathbf{d}_{\beta}\right| \xi_{\alpha \beta}\left(\omega_{a}, \omega_{b}\right)\right\rangle
\end{aligned}
$$

where $\mathbf{d}$ is the dipole operator defined previously, $E_{0}$ the ground-state energy, $H_{0}$ the unperturbed Hamiltonian [defined in Eq. (90] and $\left|\psi_{\alpha}\right\rangle$ the basis obtained via the $\mathbf{c}^{ \pm}$coefficients and the Bessel functions $J_{1}\left(z_{1, n}\right)$ in Eq. 
(17). This functional is then minimized with respect to $\left|\xi_{\alpha \beta}\right\rangle$, defined analogously to Eq. (17) as

$$
\begin{aligned}
& \xi_{\alpha \beta}\left(\omega_{a}, \omega_{b} ; r\right)= \\
& =\sum_{n=1}^{+\infty} \sum_{|l|=0}^{+\infty} \zeta_{l, n}^{\alpha, \beta}\left(\omega_{a}, \omega_{b}\right) J_{l}\left(z_{l, n} \frac{r}{R}\right) \frac{e^{i l \theta}}{\sqrt{2 \pi}}
\end{aligned}
$$

where we will focus our attention on the $x x$ component of $\xi_{\alpha \beta}$.

The discussion of minimization of this $\mathcal{K}$ functional, done in a similar fashion as for the $\mathcal{J}$ functional, is performed in-depth in Appendix C

Having defined both $\zeta_{0}^{x, x}$ and $\zeta_{2}^{x, x}$ in Eqs. C9 $\mathrm{C} 12$, respectively, the third-order susceptibility follows from [49] (with $\omega_{\sigma}=\omega_{1}+\omega_{2}+\omega_{3}$ ) as

$$
\begin{aligned}
\chi_{x, x, x, x}^{(3)}\left(-\omega_{\sigma} ; \omega_{1}, \omega_{2}, \omega_{3}\right) & =\frac{1}{3 !} \mathcal{P}\left\{-\mathbf{c}^{\dagger}\left(\left(-\omega_{\sigma}\right)^{\dagger}\right) \cdot\left[\mathbb{T}_{0} \cdot \zeta_{0}^{x, x}\left(-\omega_{2},-\omega_{3}\right)+\mathbb{T}_{2} \cdot \zeta_{2}^{x, x}\left(-\omega_{2},-\omega_{3}\right)\right]+\right. \\
& \left.+\left[\mathbf{S} \cdot \mathbf{c}\left(-\omega_{\sigma}\right)\right] \times\left[\sum_{n=1}^{N} c_{n}^{\dagger}\left(\left(-\omega_{2}\right)^{\dagger}\right) c_{n}\left(\omega_{1}\right) 2 \pi \frac{R^{2}}{2}\left[J_{2}\left(\mathrm{z}_{1, n}\right)\right]^{2}\right]\right\},
\end{aligned}
$$

with $\mathcal{P}$ denoting the different permutations of the frequencies $\left(-\omega_{\sigma} ; \omega_{1}, \omega_{2}, \omega_{3}\right), \mathbb{T}_{0 / 2}$ is defined in Eq. C3, and $\mathbf{S}$ is the vector in Eq. A4). Having defined Eq. 22, we will now utilize it to compute two different regimes for the third-order polarizability of interlayer excitons in $\mathrm{WSe}_{2} / \mathrm{WS}_{2}$.

\section{A. Two-Photon Absorption in $\mathrm{WSe}_{2} / \mathrm{WS}_{2}$}

We will now compute the $x x x x$ component of the twophoton absorption third-order susceptibility, defined as $\chi_{x x x x}^{\mathrm{TPA}}(\omega)=\chi_{x x x x}^{(3)}(-\omega ; \omega,-\omega, \omega)$.

As the two-photon absorption was considered in [49], we will not discuss it as in-depth as we will with the third-harmonic generation. Similarly to what was done in Fig. 33, we plot the real and imaginary parts of the two-photon absorption third-order susceptibility for a broadening small enough $(0.05 \mathrm{meV})$ such that each individual peak can be clearly observed. This plot is visible in Fig. 4 normalized by the peak value for the $1 \rightarrow 3 d$ transition at $\sim 16.6 \mathrm{meV}$, along with a diagram representing the $1 s \rightarrow n s$ and $1 s \rightarrow n d$ transitions.

\section{B. Third-Harmonic Generation}

To finalize this paper, we will now compute the $x x x x$ component of the third-order susceptibility thirdharmonic generation of interlayer excitons in $\mathrm{WSe}_{2} / \mathrm{WS}_{2}$, which is defined as $\chi_{x x x x}^{\mathrm{THG}}(3 \omega)=\chi_{x x x x}^{(3)}(-3 \omega ; \omega, \omega, \omega)$. Following the same approach as in Sec. IVA, we will begin by computing $\chi_{x x x x}^{\mathrm{THG}}(3 \omega)$ for a small enough broadening such that each resonance is clear.

The left-hand side of Fig. 5 has been normalized by the peak value for the resonance at $3 \hbar \omega=E_{1 s \rightarrow 2 p}$, whilst the right-hand side features a diagram representing the various transitions.
Spectral broadening as low as $\Delta \sim 2 \mathrm{meV}$ (i.e., the broadening that was considered for Fig. 33 can be achieved for low temperature encapsulated systems [64. Considering much higher values of the broadening than in Fig. 5 namely $\Delta=1 \mathrm{meV}$ and $\Delta=2 \mathrm{meV}$, we recompute $\chi_{x x x x}^{\mathrm{HHG}}(3 \omega)$, obtaining Fig. 6

Although some $1 s \rightarrow n p$ resonances are already apparent at $\Delta=2 \mathrm{meV}$, namely at $\sim 40 \mathrm{meV}(1 s \rightarrow 5 p)$ and $\sim 60 \mathrm{meV}(1 s \rightarrow 8 p)$, most $1 s \rightarrow n s$ and $1 s \rightarrow n d$ transitions only become clearly visible in the sub-meV regime. At those broadenings, the most clear peaks are at $3 \hbar \omega \approx 50 \mathrm{meV}(1 s \rightarrow 3 d)$ and at $3 \hbar \omega \approx 56 \mathrm{meV}$ $(1 s \rightarrow 4 s)$. The two resonances at $\sim 72 \mathrm{mev}$ and $\sim 75 \mathrm{mev}$ are joined into one at $\Delta=1 \mathrm{meV}$, although their presence is noticeable from comparison with the adjacent $1 s \rightarrow n p$ transition peaks.

The $1 s \rightarrow n p$ resonance peaks occur for $3 \hbar \omega=E_{1 s \rightarrow n p}$, while the $1 s \rightarrow n s$ and $1 s \rightarrow n d$ peaks are situated at $3 \hbar \omega=\frac{3}{2} E_{1 s \rightarrow n s / n d}$. Additionally, the peak at $3 \hbar \omega=$ $87.1727 \mathrm{meV}$ corresponds to $3 \hbar \omega=3 E_{1 s \rightarrow 2 p}$. This is due to the response at both $2 \omega$ [65] and $\omega$ also being present in this process, described in detail in Section 3.2 of [66], i.e. $2 \hbar \omega=E_{1 s \rightarrow n s / n d}$ and $\hbar \omega=E_{1 s \rightarrow 2 p}$. No other peaks for $\hbar \omega=E_{1 s \rightarrow n p}$ are visible, as they would already be outside the considered frequency domain $\left(3 E_{1 s \rightarrow 3 p} \sim\right.$ $105.872 \mathrm{meV}$, barely outside of the plot).

\section{CONCLUSIONS}

In this paper, we discussed interlayer excitons in a $\mathrm{WSe}_{2} / \mathrm{WS}_{2}$-based van der Waals heterostructure, for which recent experimental measurements of the polarizability through pump-probe experiments [21] have been performed. In these experiments, the transition from bright to dark states has been accessed for both interlayer and intralayer excitons. In this paper, we focused ourselves solely on the transitions between intra-excitonic 


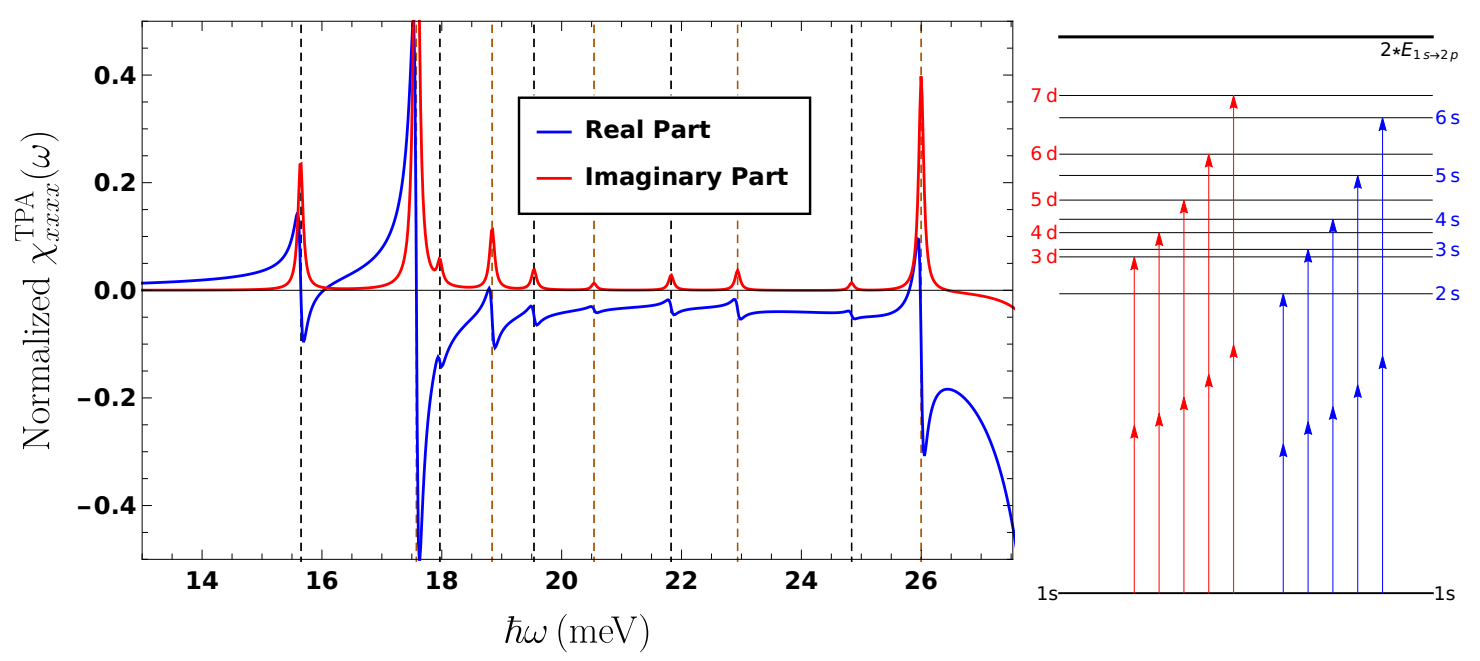

Figure 4.

Left: Real (blue) and imaginary (red) parts of the two-photon absorption third-order susceptibility (normalized by the peak value for the $1 \rightarrow 3 d$ transition at $\sim 17.5 \mathrm{meV}$ ) as a function of the incident photons' energies for a broadening $\Delta=0.05 \mathrm{meV}$ and basis size $N=120$. The resonances correspond to $1 s \rightarrow n s$ transitions (black dashed lines) and to $1 s \rightarrow n d$ transitions (dark-orange dashed lines). The dominant $1 s \rightarrow 2 p$ transition is at $\sim 29.0576 \mathrm{meV}$, outside of the frequency range plotted in this figure. Right: Diagramatic representation of the $1 s \rightarrow n s$ (blue arrows) and $1 s \rightarrow n d$ (red arrows) transitions. Topmost black line represents the resonance when $\hbar \omega=E_{1 s \rightarrow 2 p}$. We remark that the red and blue arrows represent the selection rules of the system, that is, the only allowed transitions.

states for interlayer excitons in a circular $\mathrm{WSe}_{2} / \mathrm{WS}_{2}$ dot.

Approximating the interlayer modified RytovaKeldysh potential in a power series, we motivate a Gaussian variational ansatz for the ground state of the system. After discussing the necessary material-dependent parameters, we consider both this ansatz and a complete set of basis functions the Bessel functions of the first kind to estimate the excitonic wave functions through variational methods.

We apply Fowler's and Karplus' method variational method to access the linear polarizability of twodimensional interlayer excitons, focusing on the transition from bright to dark states (more specifically, $1 s \rightarrow$ $n p$ transitions) in a circular dot of a $\mathrm{WSe}_{2} / \mathrm{WS}_{2}$-based van der Waals heterostructure with Dirichlet boundary conditions.

For the linear regime we consider a broadening of $\Delta=2 \mathrm{meV}$, allowing for the clear observation of the multiple peaks in the excitonic polarizability. We observe an almost perfect agreement between the frequency of each resonance and the energy differences between the ground state and the excited states of the exciton. These energy differences were calculated both numerically (via the Numerov shooting method) and variationally (via both a Gaussian ansatz for the ground-state and a finite basis of Bessel function of the first kind), allowing for a greater confidence in their values. As expected 21, comparing the linear polarizability of interlayer excitons in the hetero-bilayer against intralayer excitons in each of the individual layers shows that the binding energies are much lower in the interlayer regime (Figs. 3 and 7 . Table II and Eq. (B2)).
Onto the third-order polarizability, we began by providing an in-depth discussion of Svendsen's variational method [47, 48]. After outlining this method and analyzing the required modifications due to the necessity of orthogonality between the various states, we work on the algebraization of the method, arriving at a purely vectorial problem after the necessary integrals are computed. The obtained expression is then tested first with the twophoton absorption, and then with third-harmonic generation.

Starting by two-photon absorption, a process which was discussed in-depth by Henriques et al. [49, we perform the necessary calculations with a broadening small enough such that the resonance associated with each individual intra-excitonic energy level transition can be clearly identifiable $(\Delta=0.05 \mathrm{meV})$. The two-photon absorption third-order susceptibility is then normalized by the value at the $E_{1 s \rightarrow 3 d}$ resonance as to facilitate the comparison of each peak. Of note is the amplitude of the resonance associated with the $E_{1 s \rightarrow 7 d}$ transition, peaking at around $40 \%$ of the maximum and being dramatically larger than the adjacent transitions. Additionally, the individual selection rules for this system were identified.

Finally turning to the third-harmonic generation in the third-order susceptibility, we first perform the calculations for a very small broadening $(\Delta=0.05 \mathrm{meV})$. Each individual transition was clearly identifiable, with resonances at $\hbar \omega=E_{i \rightarrow f}$ and $3 \hbar \omega=E_{i \rightarrow f}$ for $1 s \rightarrow n p$ transitions, and at $2 \hbar \omega=E_{i \rightarrow f}$ for both $1 s \rightarrow n s$ and $1 s \rightarrow n d$ transitions. The individual selection rules for the third-harmonic generation process were clearly identifiable. 


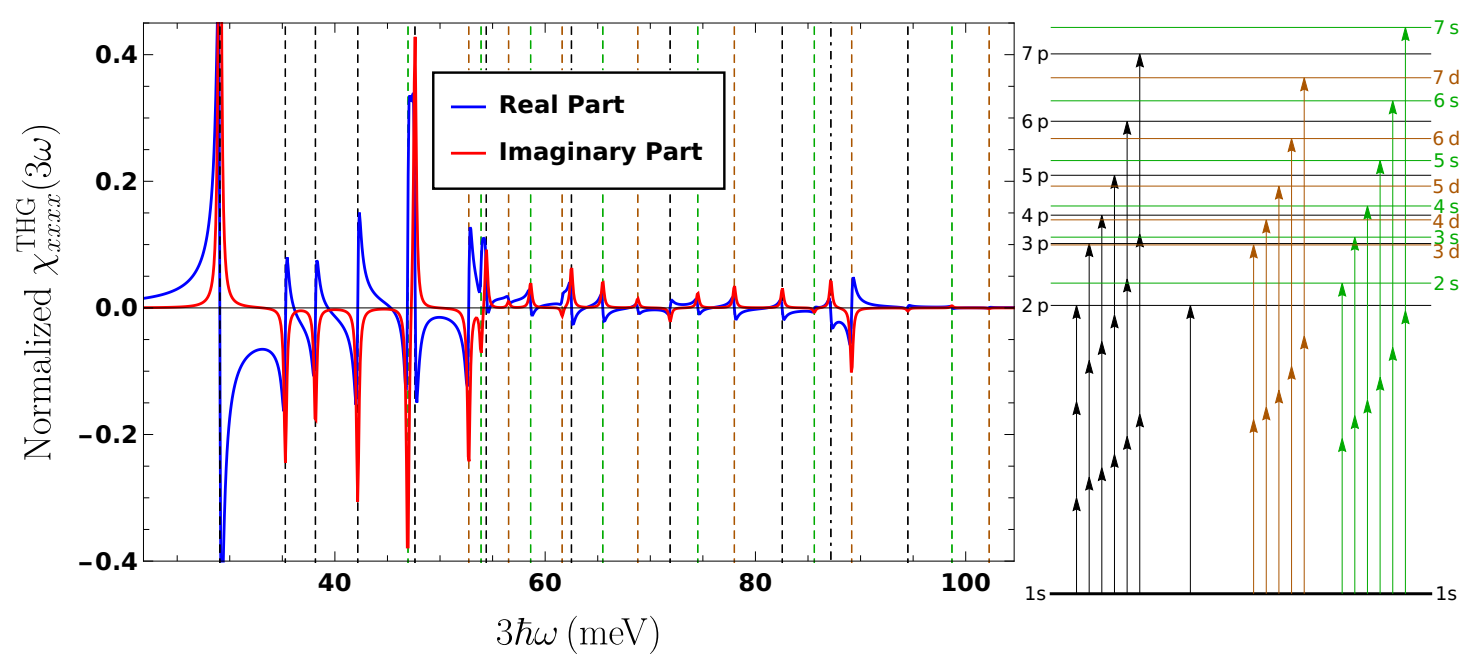

Figure 5.

Left: Real (blue) and imaginary (red) parts of the third-harmonic generation third-order susceptibility (normalized by the peak value for the $1 \rightarrow 2 p$ resonance at $\sim 29.0576, \mathrm{meV})$ as a function of the incident photons' energies for a broadening $\Delta=0.05 \mathrm{meV}$ and basis size $N=120$. The resonances correspond to $1 s \rightarrow n p$ transitions (black dashed lines), $1 s \rightarrow n s$ transitions (green dashed lines) and $1 s \rightarrow n d$ transitions (dark-orange dashed lines). The $\sim 87.1727$ meV resonance (black dot-dashed line) corresponds to the response at frequency $3 \hbar \omega=3 E_{1 s \rightarrow 2 p}$. Right: Diagramatic representation of the various transitions visible in the plot on the left-hand side. The colors of the arrows represent the different transitions: $1 s \rightarrow n p$ (black), $1 s \rightarrow n s$ (green), and $1 s \rightarrow n d$ (dark-orange), while the number of arrows represents the frequency of the transition: 3 arrows for $3 \hbar \omega=E_{i \rightarrow f}, 2$ arrows for $2 \hbar \omega=E_{i \rightarrow f}$, and 1 arrow for $\hbar \omega=E_{i \rightarrow f}$. Again, as in Fig. 4 the arrows represent the selection rules associated with the third-harmonic generation (THG) nonlinear process.
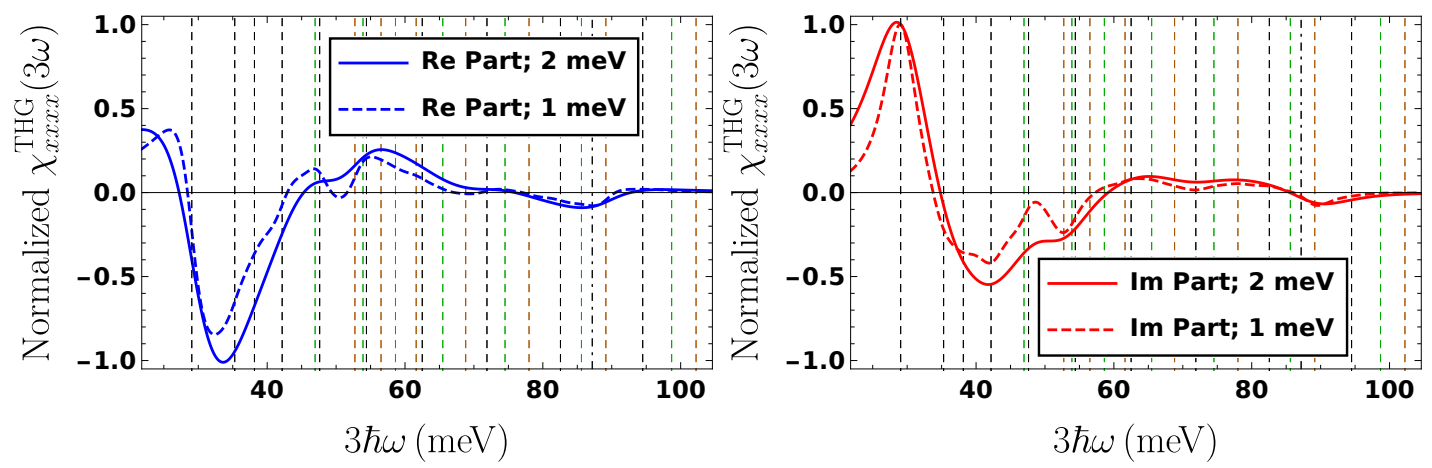

Figure 6. Real (left) and imaginary (right) parts of the normalized third-harmonic generation third-order susceptibility for different broadening values: $\Delta=2 \mathrm{meV}$ (solid lines) and $1 \mathrm{meV}$ (dashed lines). The basis size is fixed at $N=120$. The resonances correspond to $1 s \rightarrow n p$ transitions (black dashed lines), $1 s \rightarrow n s$ transitions (green dashed lines) and $1 s \rightarrow n d$ transitions (dark-orange dashed lines). The $\sim 87.1727 \mathrm{meV}$ resonance (black dot-dashed line) corresponds to the response at $3 \hbar \omega=3 E_{1 s \rightarrow 2 p}$.

Considering experimentally-obtainable values of the broadening, we compare the third-harmonic generation third-order susceptibility for $\Delta=1 \mathrm{meV}$ and $2 \mathrm{meV}$. We argue for the feasibility of experimentally observing the three well-resolved resonance peaks, associated with $1 s \rightarrow 2 p, 1 s \rightarrow 3 d$, and $1 s \rightarrow 4 s$ transitions, where the latter two could be an experimental indication of thirdharmonic generation due to intra-excitonic transitions in this specific van der Waals heterostructure.

\section{ACKNOWLEDGMENTS}

M.F.C.M.Q. acknowledges the International Nanotechnology Laboratory for the Quantum Portugal Initiative grant $\mathrm{SFRH} / \mathrm{BD} / 151114 / 2021$. N.M.R.P acknowledges support by the Portuguese Foundation for Science and Technology (FCT) in the framework of the Strategic Funding UIDB $/ 04650 / 2020$. J.C.G.H. acknowledges the Center of Physics for a grant funded by the UIDB $/ 04650 / 2020$ strategic project. N.M.R.P. also acknowledges support from the European Com- 
mission through the project "Graphene-Driven Revolutions in ICT and Beyond" (Ref. No. 881603, CORE 3), COMPETE 2020, PORTUGAL 2020, FEDER and the FCT through projects POCI-01-0145-FEDER028114, POCI-01-0145-FEDER-02888 and PTDC/NAN$\mathrm{OPT} / 29265 / 2017$.

\section{Appendix A: Variational Coefficients for the Linear Polarizability}

Recalling the orthogonality relation of Bessel functions on a finite disk of radius $R$ [67],

$$
\begin{array}{r}
\int_{0}^{R} J_{\nu}\left(\frac{z_{v m} r}{R}\right) J_{\nu}\left(\frac{z_{v n} r}{R}\right) r d r= \\
=\frac{R^{2}}{2} \delta_{n m}\left[J_{|\nu|+1}\left(z_{v m}\right)\right]^{2}
\end{array}
$$

with $z_{v m}$ the $m$-th zero of $J_{\nu}(z)$, one easily shows that the functional can be rewritten as

$$
\begin{aligned}
& \mathcal{J}_{ \pm}=\pi \sum_{n=1} \sum_{k=1} c_{n}^{ \pm} c_{k}^{ \pm} \mathcal{I}_{k n}+2 \pi \sum_{n=1} c_{n}^{ \pm} \mathcal{S}_{n}+ \\
& +\frac{\pi R^{2}}{2} \sum_{n=1}\left(c_{n}^{ \pm}\right)^{2}\left[\frac{\hbar^{2}}{2 \mu} \frac{z_{1 n}^{2}}{R^{2}}-E_{0} \pm \hbar \omega\right]\left[J_{2}\left(z_{1 n}\right)\right]^{2}
\end{aligned}
$$

where $\mathcal{I}_{k n}$ and $\mathcal{S}_{n}$ refer to the following integrals involving two and one Bessel functions, respectively

$$
\begin{gathered}
\mathcal{I}_{k n}=\int_{0}^{R} J_{1}\left(\frac{z_{1 k} r}{R}\right) V_{\mathrm{RK}}(r) J_{1}\left(\frac{z_{1 n} r}{R}\right) r d r, \\
\mathcal{S}_{n}=\int_{0}^{R} J_{1}\left(\frac{z_{1 n} r}{R}\right) r \psi_{0}^{\mathrm{fin} .}(r) r d r .
\end{gathered}
$$

Differentiating $\mathcal{J}_{ \pm}$with respect to the different $c_{n}^{ \pm}$coefficients, one finds

$$
\begin{aligned}
& c_{j}^{ \pm}\left\{\frac{R^{2}\left[J_{2}\left(z_{1 j}\right)\right]^{2}}{2}\left[\frac{z_{1 j}^{2}}{2 \mu R^{2}}-E_{0} \pm \omega\right]+\mathcal{I}_{j j}\right\}+ \\
& +\sum_{n \neq j}^{N} c_{n}^{ \pm} \mathcal{I}_{j n}=-\mathcal{S}_{j},
\end{aligned}
$$

with $j \in\{1,2, \ldots, N\}$. This equation defines a linear system of equations whose solution determines the values of the coefficients $c_{n}^{ \pm}$.

We can write Eq. A5 in a more concise manner, using matrix notation, as

$$
\mathbb{M} \cdot \mathbf{c}^{ \pm}=-\mathbf{S},
$$

where $\mathbf{c}^{ \pm}$and $\mathbf{S}$ are column vectors defined as

$$
\begin{aligned}
{\left[\mathbf{c}^{ \pm}\right]^{\mathrm{T}} } & =\left(c_{1}^{ \pm}, c_{2}^{ \pm}, \ldots, c_{N}^{ \pm}\right), \\
\mathbf{S}^{\mathrm{T}} & =\left(\mathcal{S}_{1}, \mathcal{S}_{2}, \ldots, \mathcal{S}_{N}\right),
\end{aligned}
$$

and $\mathbb{M}$ is a $N \times N$ matrix with:

$$
(\mathbb{M})_{i j}=g_{i}^{ \pm}(\omega) \delta_{i j}+\mathcal{I}_{i j},
$$

where $\delta_{i j}$ is the Kronecker delta and

$$
g_{i}^{ \pm}(\omega)=\frac{R^{2}\left[J_{2}\left(z_{1 i}\right)\right]^{2}}{2}\left[\frac{z_{1 i}^{2}}{2 \mu R^{2}}-E_{0} \pm \omega\right] .
$$

After $\mathbb{M}$ and $\mathbf{S}$ are computed using Eqs. A3 and A9, and (A4), respectively, the coefficients that determine $\psi_{1}^{ \pm}(\mathbf{r})$ are readily obtained as

$$
\mathbf{c}^{ \pm}=-\mathbb{M}^{-1} \cdot \mathbf{S}
$$

and the solution of the differential equation is found.

\section{Appendix B: Linear Polarizability for interlayer and intralayer excitons}

In this appendix we will make a quick comparison between the linear polarizability of both interlayer and intralayer excitons in a $\mathrm{WSe}_{2} / \mathrm{WS}_{2}$ heterobilayer.

Intralayer excitons are considered for both the $\mathrm{WSe}_{2}$ and the $\mathrm{WS}_{2}$ layers, with the relevant material-dependent parameters. Additionally, due to the Coulomb-like divergence of the potential at $r=0$, we consider the more appropriate variational ansatz, defined by Pedersen [55], given by

$$
\psi_{0}(r)=\mathcal{C}\left(e^{-a r}-b e^{-\gamma a r}\right)(R-r) .
$$

Minimizing this functional, the ground-state energy for excitons each layer will be

$$
\begin{aligned}
E_{\mathrm{WS}_{2}} & =-0.00438405=-119.296 \mathrm{meV}, \\
E_{\mathrm{WSe}_{2}} & =-0.00541680=-147.399 \mathrm{meV},
\end{aligned}
$$

both closely matching the energies obtained via the finite basis approach from Sec IIC. These binding energies are also substantially larger than the ground-state energy for interlayer excitons in the heterobilayer (Table II), as expected from experimental studies [21].

Computing the linear polarizability with the formalism described in Appendix A normalized by the value at the $1 s \rightarrow 2 p$ peak, we display the results in Fig. 7 .

\section{Appendix C: Variational Coefficients for the Third-Order Polarizability}

Defining $g_{n}^{(l)}(\omega)$ as a more general form of Eq. A9

$$
g_{n}^{(l)}(\omega)=\frac{R^{2}\left[J_{|l|+1}\left(z_{l, n}\right)\right]^{2}}{2}\left[\frac{z_{l, n}^{2}}{2 \mu R^{2}}-E_{0}+\omega\right]
$$




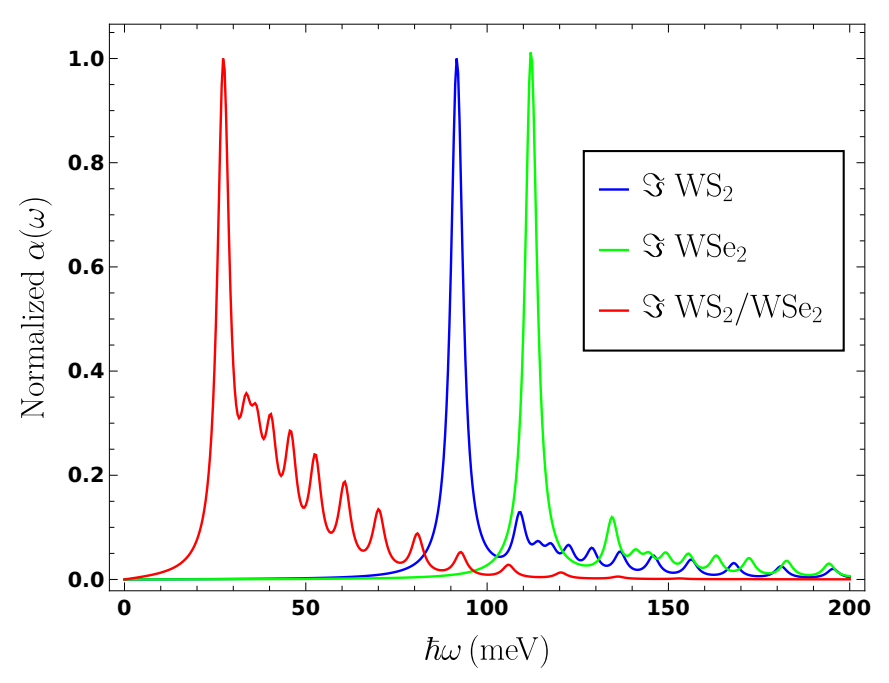

Figure 7. Imaginary part of the normalized linear polarizability for interlayer excitons in a $\mathrm{WSe}_{2} / \mathrm{WS}_{2}$ heterostructure and intralayer excitons in both a $\mathrm{WS}_{2}$ and a $\mathrm{WSe}_{2}$ monolayers. The peaks correspond to transitions between the $1 s$ state (ground-state) and the excited $n p$ states.

and $\mathcal{V}_{n m}^{(l)}$ analogously to Eq. A3

$$
\begin{aligned}
\mathcal{V}_{n m}^{(l)} & =\left\langle J_{l}\left(z_{l, n} \frac{r}{R}\right)\left|V_{i-R K}(r)\right| J_{l}\left(z_{l, m} \frac{r}{R}\right)\right\rangle \\
& =\int_{0}^{R} J_{l}\left(z_{l, n} \frac{r}{R}\right) V_{i-R K} J_{l}\left(z_{l, m} \frac{r}{R}\right) r d r
\end{aligned}
$$

the functional of Eq. 20 can then be rewritten using the both the $c_{n}^{ \pm}$and the $\zeta_{l, n}$ coefficients as

$$
\begin{aligned}
\mathcal{K}= & \sum_{n=1}^{+\infty} \sum_{|l|=0}^{+\infty}\left[\zeta_{l, n}\right]^{\dagger} g_{n}^{(l)}\left(\omega_{a}+\omega_{b}\right) \zeta_{l, n}+ \\
& +\sum_{n, m=1}^{+\infty} \sum_{|l|=0}^{+\infty}\left[\zeta_{l, n}\right]^{\dagger} \mathcal{V}_{n m}^{(l)} \zeta_{l, m}+ \\
& +\frac{1}{2} \sum_{n, m=1}^{+\infty}\left\{\left[\zeta_{0, n}\right]^{\dagger} \mathcal{T}_{n m}^{l=0} \cdot\left(c_{m}^{+}+c_{m}^{-}\right)+\right. \\
& \left.+\left[\zeta_{|l|=2, n}\right]^{\dagger}\left(\mathcal{T}_{n m}^{l=2} \cdot c_{m}^{+}+\mathcal{T}_{n m}^{l=-2} \cdot c_{m}^{-}\right)+c . c .\right\}
\end{aligned}
$$

In Eq. C1, $\mathcal{T}_{n m}^{l}$ is the dipole transition amplitude between the functions of the basis, denoted by $\left\langle\psi_{\alpha}\left(\omega_{a}\right)\left|\mathbf{d}_{\beta}\right| \xi_{\alpha \beta}\left(\omega_{a}, \omega_{b}\right)\right\rangle$ in Eq. 20 and given by

$$
\begin{aligned}
\mathcal{T}_{n m}^{l} & =\left\langle J_{l}\left(z_{l, n} \frac{r}{R}\right)|r| J_{1}\left(z_{1, m} \frac{r}{R}\right)\right\rangle \\
& =\int_{0}^{R} J_{l}\left(z_{l, n} \frac{r}{R}\right) r J_{1}\left(z_{1, m} \frac{r}{R}\right) r d r .
\end{aligned}
$$

The solution of this integral can be written in a closed form by applying the more general expression

$$
\begin{gathered}
\int_{0}^{1} J_{\nu}(\alpha r) r J_{\nu+1}(\beta r) r d r=\frac{\alpha J_{\nu+1}(\alpha)}{\left(\alpha^{2}-\beta^{2}\right)^{2}} \times \\
\times\left[-2 \beta J_{\nu}(\beta)+\left(\alpha^{2}-\beta^{2}\right) J_{\nu+1}(\beta)\right],
\end{gathered}
$$

valid as long as $J_{\nu}(\alpha)=0$. Comparing with the definition of $\mathcal{T}_{n m}^{l}$, Eq. C2 can be simplified further as $J_{\nu+1}(\beta)=0$. Performing the necessary changes of variable, and substituting $J_{\nu+1}(\beta)$ into Eq. $(\mathrm{C} 2), \mathcal{T}_{n m}^{l}$ can be written as

$$
\begin{aligned}
& \mathcal{T}_{n m}^{(l=0)}=-2 R^{3} \frac{z_{0, n} z_{1, m} J_{1}\left(z_{0, n}\right) J_{0}\left(z_{1, m}\right)}{\left(z_{0, n}^{2}-z_{1, m}^{2}\right)^{2}} \\
& \mathcal{T}_{n m}^{(l=2)}=-2 R^{3} \frac{z_{1, n} z_{2, m} J_{2}\left(z_{1, n}\right) J_{1}\left(z_{2, m}\right)}{\left(z_{1, n}^{2}-z_{2, m}^{2}\right)^{2}}
\end{aligned}
$$

As done in the appendix of [49, we separate the $l=0$ and the $l=2$ cases. Looking first at the more complicated case of $l=0$, we must ensure that $\left|\xi_{\alpha \beta}\right\rangle$ is orthogonal to the considered ground-state [(in our case, the ansatz of Eq. [8] ]. As such, to ensure this, we must have

$$
\begin{aligned}
\left\langle\psi_{0} \mid \xi_{\alpha \beta}\right\rangle & =\sum_{n=1}^{+\infty} \zeta_{0, n}^{\alpha, \beta}\left(\omega_{a}, \omega_{b}\right)\left\langle\psi_{0} \mid J_{0}\left(z_{0, n} \frac{r}{R}\right)\right\rangle \\
& =0
\end{aligned}
$$

where non-zero angular momentum terms vanish upon angular integration due to the isotropic nature of the ground-state ansatz. Separating the first term of the sum, this condition is given by

$$
\begin{aligned}
& \zeta_{0,1}^{\alpha, \beta}\left(\omega_{a}, \omega_{b}\right)\left\langle\psi_{0} \mid J_{0}\left(z_{0,1} \frac{r}{R}\right)\right\rangle+ \\
& +\sum_{n=2}^{+\infty} \zeta_{0, n}^{\alpha, \beta}\left(\omega_{a}, \omega_{b}\right)\left\langle\psi_{0} \mid J_{0}\left(z_{0, n} \frac{r}{R}\right)\right\rangle=0,
\end{aligned}
$$

which can then be rewritten as

$$
\begin{aligned}
& \zeta_{0,1}^{\alpha, \beta}\left(\omega_{a}, \omega_{b}\right)= \\
& =-\sum_{n=2}^{+\infty} \zeta_{0, n}^{\alpha, \beta}\left(\omega_{a}, \omega_{b}\right) \frac{\left\langle\psi_{0} \mid J_{0}\left(z_{0, n} \frac{r}{R}\right)\right\rangle}{\left\langle\psi_{0} \mid J_{0}\left(z_{0,1} \frac{r}{R}\right)\right\rangle} .
\end{aligned}
$$

This means that $\zeta_{0,1}$ is no longer considered an independent variable and, as such, we will focus our attention on the $n \geq 2$ terms. For compactness, we define

$$
\mathbf{f}_{n}=\frac{\left\langle\psi_{0} \mid J_{0}\left(z_{0, n} \frac{r}{R}\right)\right\rangle}{\left\langle\psi_{0} \mid J_{0}\left(z_{0,1} \frac{r}{R}\right)\right\rangle}
$$

which lets us rewrite Eq. C5 as

$$
\zeta_{0,1}^{\alpha, \beta}\left(\omega_{a}, \omega_{b}\right)=-\sum_{n=2}^{+\infty} \zeta_{0, n}^{\alpha, \beta}\left(\omega_{a}, \omega_{b}\right) \mathbf{f}_{n} .
$$

Substituting Eq. (C7) into Eq. (C1) and then differentiating the resulting expression with respect to $\left[\zeta_{l, n}\right]^{\dagger}$, we obtain for $n \geq 2$, 


$$
\begin{aligned}
{\left[g_{1}^{(0)}\left(\omega_{a}+\omega_{b}\right) \mathbf{f}_{n} \mathbf{f}_{m}+\mathcal{V}_{1,1}^{(0)} \mathbf{f}_{n} \mathbf{f}_{m}\right] \zeta_{0, n}-\mathcal{V}_{1, m}^{(0)} \mathbf{f}_{n} \zeta_{0, n}-\mathcal{V}_{n, 1}^{(0)} \mathbf{f}_{m} \zeta_{0, n}-\frac{1}{2} \mathbf{f}_{n} \sum_{m=1}^{+\infty} \mathcal{T}_{n m}^{l=0} \cdot\left(c_{m}^{+}+c_{m}^{-}\right)+} \\
+\sum_{n=2}^{+\infty} g_{n}^{(0)}\left(\omega_{a}+\omega_{b}\right) \zeta_{0, n}+\sum_{n, m=2}^{+\infty} \mathcal{V}_{n m}^{(0)} \zeta_{0, m}+\frac{1}{2} \sum_{n, m=2}^{+\infty}\left\{\mathcal{T}_{n m}^{l=0} \cdot\left(c_{m}^{+}+c_{m}^{-}\right)+c . c .\right\}=0
\end{aligned}
$$

This means that the system can be easily solved algebraically as

$$
\zeta_{0}^{x, x}\left(\omega_{a}, \omega_{b}\right)=\left[\mathbb{F}+\mathbb{M}^{(0)}\left(\omega_{a}+\omega_{b}\right)\right]^{-1} \cdot\left[\mathbf{W}_{0}^{x, x}\left(\omega_{a}\right)+\mathbf{f}_{0}^{x, x}\left(\omega_{a}\right)\right]
$$

with $\mathbb{M}^{(0)}\left(\omega_{a}+\omega_{b}\right)$ defined as in Eq. A7 and

$$
\begin{gathered}
\mathbf{W}_{0}^{x, x}=-\mathbb{T}^{0} \cdot \mathbf{c} \\
\left(\mathbf{f}_{0}^{x, x}\right)_{n}=\mathbf{f}_{n} \sum_{m=1}^{N} c_{m} \mathcal{T}_{1, m}^{(0)} c_{m}, \\
(\mathbb{F})_{i, j}=\left[g_{1}^{(0)}\left(\omega_{a}+\omega_{b}\right)+\mathcal{V}_{1,1}^{(0)}\right] \mathbf{f}_{i} \mathbf{f}_{j}- \\
-\mathcal{V}_{1, j}^{(0)} \mathbf{f}_{i}-\mathcal{V}_{i, 1}^{(0)} \mathbf{f}_{j} .
\end{gathered}
$$

The missing coefficient $\zeta_{0,1}^{\alpha, \beta}\left(\omega_{a}, \omega_{b}\right)$ is obtained by calculating Eq. C7 with the solution of Eq. C9. Several expressions have been already simplified by taking advantage of the parity of Bessel functions of the first kind,

$$
J_{-l}(x)=(-1)^{l} J_{l}(x)
$$

Looking now at the simpler $l=2$ case, and as no orthogonality-based restrictions need to be applied to its coefficients, we can directly differentiate Eq. (C1) with respect to $\left[\zeta_{l, n}\right]^{\dagger}$ and then minimize the resulting expression, obtaining

$$
\begin{aligned}
& \sum_{n=1}^{+\infty} g_{n}^{(2)}\left(\omega_{a}+\omega_{b}\right) \zeta_{2, n}+\sum_{n, m=1}^{+\infty} \mathcal{V}_{n m}^{(2)} \zeta_{2, m}+ \\
& +\frac{1}{2} \sum_{n, m=1}^{+\infty}\left\{\mathcal{T}_{n m}^{l=2} \cdot c_{m}^{+}+\mathcal{T}_{n m}^{l=-2} \cdot c_{m}^{-}\right\}=0
\end{aligned}
$$

The $\zeta_{2, n}$ variational coefficients can then be obtained, in vector form, as

$$
\zeta_{2}^{x, x}\left(\omega_{a}, \omega_{b}\right)=\left[\mathbb{M}^{(2)}\left(\omega_{a}+\omega_{b}\right)\right]^{-1} \cdot \mathbf{W}_{2}^{x, x}\left(\omega_{a}\right),
$$

a similar system to the one in Eq. A10 with $\mathbf{W}_{2}^{x, x}$ defined analogously to $\mathbf{W}_{0}^{x, x}$, after simplifications from parity of Bessel functions.
[1] N. Mounet, M. Gibertini, P. Schwaller, D. Campi, A. Merkys, A. Marrazzo, T. Sohier, I. E. Castelli, A. Cepellotti, G. Pizzi, and N. Marzari. Two-dimensional materials from high-throughput computational exfoliation of experimentally known compounds. Nat. Nano., 13(3):246-252, feb 2018.

[2] R. F. Frindt and A. D. Yoffe. Phys. properties of layer structures : optical properties and photoconductivity of thin crystals of molybdenum disulphide. Proc. R. Soc. Lond. A, 273(1352):69-83, apr 1963.

[3] E. Fortin and W.M. Sears. Photovoltaic effect and optical absorption in MoS2. J. of Phys. and Chem. of Sol., 43(9):881-884, jan 1982.

[4] R. Lv, J. A. Robinson, R. E. Schaak, D. Sun, Y. Sun, T. E. Mallouk, and M. Terrones. Transition Metal Dichalcogenides and Beyond: Synthesis, Properties, and Applications of Single- and Few-Layer Nanosheets. Acc. of Chem. Res., 48(1):56-64, 2015.

[5] T. Mueller and E. Malic. Exciton physics and device application of two-dimensional transition metal dichalco- genide semiconductors. npj 2D Mat. and App., 2(1):29, December 2018.

[6] G. Wang, A. Chernikov, M. M. Glazov, T. F. Heinz, X. Marie, T. Amand, and B. Urbaszek. Colloquium: Excitons in atomically thin transition metal dichalcogenides. Rev. Mod. Phys., 90(2):021001, 2018.

[7] Q. Ma, G. Ren, K. Xu, and J. Z. Ou. Tunable Optical Properties of 2D Materials and Their Applications. Adv. Opt. Mat., page 2001313, 2020.

[8] H. Yu, X. Cui, X. Xu, and W. Yao. Valley excitons in two-dimensional semiconductors. Nat. Sci. Rev., 2(1):57$70,2015$.

[9] C. Schneider, M. M. Glazov, T. Korn, S. Höfling, and B. Urbaszek. Two-dimensional semiconductors in the regime of strong light-matter coupling. Nat. Comm., 9(1):2695, 2018.

[10] W.-T. Hsu, J. Quan, C.-Y Wang, L.-S. Lu, M Campbell, W-H Chang, L.-J. Li, X. Li, and C.-K. Shih. Dielectric impact on exciton binding energy and quasiparticle bandgap in monolayer WS 2 and WSe 2. 2D Mat., 
6:025028, 2019.

[11] X.-X. Zhang, T Cao, Z Lu, Y.-C. Lin, F. Zhang, Y Wang, Z. Li, J. C. Hone, J. A. Robinson, D. Smirnov, S. G. Louie, and T. F. Heinz. Magnetic brightening and control of dark excitons in monolayer WSe 2. Nat. Nano., 12(9):883-888, 2017.

[12] A. Taghizadeh and T. G. Pedersen. Nonlinear optical selection rules of excitons in monolayer transition metal dichalcogenides. Phys. Rev. B, 99:235433, Jun 2019.

[13] M. V. Durnev and M. M. Glazov. Excitons and trions in two-dimensional semiconductors based on transition metal dichalcogenides. Physics-Uspekhi, 61(9):825-845, sep 2018.

[14] M. Koperski, M. R. Molas, A. Arora, K. Nogajewski, A. O. Slobodeniuk, C. Faugeras, and M. Potemski. Optical properties of atomically thin transition metal dichalcogenides: observations and puzzles. Nanophotonics, 6(6):1289 - 1308, 01 Nov. 2017.

[15] G. Berghäuser, A. Knorr, and E. Malic. Optical fingerprint of dark 2p-states in transition metal dichalcogenides. 2D Mat., 4(1):015029, 2016.

[16] K. Miyajima, K. Sakaniwa, and M. Sugawara. Optical transitions from the lowest to higher exciton and biexciton Rydberg states in CuCl. Phys. Rev. B, 94(19):195209, 2016. Publisher: American Physical Society.

[17] D. Fröhlich, A. Nöthe, and K. Reimann. Observation of the Resonant Optical Stark Effect in a Semiconductor. Phys. Rev. Let., 55(12):1335-1337, 1985.

[18] G. Berghäuser, P. Steinleitner, P. Merkl, R. Huber, A. Knorr, and E. Malic. Mapping of the dark exciton landscape in transition metal dichalcogenides. Phys. Rev. B, 98(2):020301, 2018.

[19] T. Tian, D. Scullion, D. Hughes, L. H. Li, C.-J. Shih, J. Coleman, M. Chhowalla, and E. J. G. Santos. Electronic Polarizability as the Fundamental Variable in the Dielectric Properties of Two-Dimensional Materials. Nano Letters, 20(2):841-851, 2020.

[20] C. Poellmann, P. Steinleitner, U. Leierseder, P. Nagler, G. Plechinger, M. Porer, R. Bratschitsch, C. Schüller, T. Korn, and R. Huber. Resonant internal quantum transitions and femtosecond radiative decay of excitons in monolayer WSe 2. Nat. Mat., 14(9):889-893, 2015.

[21] P. Merkl, F. Mooshammer, P. Steinleitner, A. Girnghuber, K.-Q. Lin, P. Nagler, J. Holler, C. Schüller, J. M. Lupton, T. Korn, S. Ovesen, S. Brem, E. Malic, and R. Huber. Ultrafast transition between exciton phases in van der waals heterostructures. Nat. Mat., 18(7):691696, April 2019.

[22] P. Rivera, K. L. Seyler, H. Yu, J. R. Schaibley, J. Yan, D. G. Mandrus, W. Yao, and X. Xu. Valley-polarized exciton dynamics in a $2 \mathrm{~d}$ semiconductor heterostructure. Science, 351(6274):688-691, February 2016.

[23] C. Jin, J. Kim, M. I. B. Utama, E. C. Regan, H. Kleemann, H. Cai, Y. Shen, M. J. Shinner, A. Sengupta, K. Watanabe, T. Taniguchi, S. Tongay, A. Zettl, and F. Wang. Imaging of pure spin-valley diffusion current in WS2-WSe2heterostructures. Science, 360(6391):893896, May 2018.

[24] Y. Gong, J. Lin, X. Wang, G. Shi, S. Lei, Z. Lin, X. Zou, G. Ye, R. Vajtai, B. I. Yakobson, H. Terrones, M. Terrones, B.-K. Tay, J. Lou, S. T. Pantelides, Z. Liu, W. Zhou, and P. M. Ajayan. Vertical and in-plane heterostructures from WS2/MoS2 monolayers. Nat. Mat., 13(12):1135-1142, September 2014.
[25] B. Miller, A. Steinhoff, B. Pano, J. Klein, F. Jahnke, A. Holleitner, and U. Wurstbauer. Long-lived direct and indirect interlayer excitons in van der Waals heterostructures. Nano Lett., 17(9):5229-5237, aug 2017.

[26] F. Vialla, M. Danovich, D. A. Ruiz-Tijerina, M. Massicotte, P. Schmidt, T. Taniguchi, K. Watanabe, R. J. Hunt, M. Szyniszewski, N. D. Drummond, T. G. Pedersen, V. I. Fal'ko, and F. H. L. Koppens. Tuning of impurity-bound interlayer complexes in a van der Waals heterobilayer. 2D Mat., 6(3):035032, may 2019.

[27] L. Chao-Cador and E. Ley-Koo. Two-dimensional hydrogen atom confined in circles, angles, and circular sectors. Int. J. of Q. Chem., 103(4):369-387, 2005.

[28] B. Munkhbat, A. B. Yankovich, D. G. Baranov, R. Verre, E. Olsson, and T. O. Shegai. Transition metal dichalcogenide metamaterials with atomic precision. Nat. Comm., 11(1):4604, 2020.

[29] F. Karimi, S. Soleimanikahnoj, and I. Knezevic. Tunable plasmon-enhanced second-order optical nonlinearity in transition metal dichalcogenide nanotriangles. Phys. Rev. B, 103:L161401, 2021.

[30] F. Wang, J. Shan, M. A. Islam, I. P. Herman, M. Bonn, and T. F. Heinz. Exciton polarizability in semiconductor nanocrystals. Nat. Mat., 5(11):861-864, 2006.

[31] S. Cha, J. H. Sung, S. Sim, J. Park, H. Heo, M.-H. Jo, and H. Choi. $1 \mathrm{~s}$-intraexcitonic dynamics in monolayer MoS 2 probed by ultrafast mid-infrared spectroscopy. Nat. Comm., 7(1):10768, 2016.

[32] D. P. Shelton. Hyperpolarizability of the hydrogen atom. Phys. Rev. A, 36(7):3032-3041, 1987.

[33] N. L. Manakov, S. I. Marmo, and E. A. Pronin. Dynamic hyperpolarizabilities of excited states of hydrogen. J. of Exp. and Theo. Phys., 98(2):254-271, February 2004.

[34] H. Zeng, G.-B. Liu, J. , Y. Yan, B. Zhu, R. He, L. Xie, S. Xu, X. Chen, W. Yao, and X. Cui. Optical signature of symmetry variations and spin-valley coupling in atomically thin tungsten dichalcogenides. Scientific Reports, 3(1), April 2013.

[35] H. G. Rosa, Y. W. Ho, I. Verzhbitskiy, M. J. F. L. Rodrigues, T. Taniguchi, K. Watanabe, G. Eda, V. M. Pereira, and J. C. V. Gomes. Characterization of the second- and third-harmonic optical susceptibilities of atomically thin tungsten diselenide. Scientific Reports, 8(1), July 2018.

[36] C. Janisch, Y. Wang, D. Ma, N. Mehta, A. L. Elías, N. Perea-López, M. Terrones, V. Crespi, and Z. Liu. Extraordinary second harmonic generation in tungsten disulfide monolayers. Scientific Reports, 4(1), July 2014.

[37] C. Torres-Torres, N. Perea-López, A. L. Elías, H. R. Gutiérrez, D. A. Cullen, A. Berkdemir, F. López-Urías, H. Terrones, and M. Terrones. Third order nonlinear optical response exhibited by mono- and few-layers of WS2. 2D Mat., 3(2):021005, April 2016.

[38] K. Yao, N. R. Finney, J. Zhang, S. L. Moore, L. Xian, N. Tancogne-Dejean, F. Liu, J. Ardelean, X. Xu, D. Halbertal, K. Watanabe, T. Taniguchi, H. Ochoa, A. AsenjoGarcia, X. Zhu, D. N. Basov, A. Rubio, C. R. Dean, J. Hone, and P. J. Schuck. Enhanced tunable second harmonic generation from twistable interfaces and vertical superlattices in boron nitride homostructures. Science Advances, 7(10):eabe8691, March 2021.

[39] V. Shahnazaryan, V. K. Kozin, I. A. Shelykh, I. V. Iorsh, and O. Kyriienko. Tunable optical nonlinearity for transition metal dichalcogenide polaritons dressed by a fermi 
sea. Phys. Rev. B, 102:115310, 2020.

[40] G. Wang, S. Zhang, X. Zhang, L. Zhang, Y. Cheng, D. Fox, H. Zhang, J. N. Coleman, W. J. Blau, and J. Wang. Tunable nonlinear refractive index of twodimensional MoS 2, WS 2, and MoSe 2 nanosheet dis-

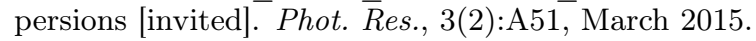

[41] S. N. Rytova. The screened potential of a point charge in a thin film. Mosc. Un. Phys. Bul., 22(30), 1967.

[42] L. V. Keldysh. Coulomb interaction in thin semiconductor and semimetal films. Sov. J. Exp. and Theor. Phys. Lett., 29:658, 1979.

[43] M. Karplus and H. J. Kolker. A Variation-Perturbation Approach to the Interaction of Radiation with Atoms and Molecules. J. of Chem. Phys., 39(6):1493-1506, 1963.

[44] P. W. Fowler. Energy, polarizability and size of confined one-electron systems. Mol. Phys., 53(4):865-889, 1984.

[45] S. Mossman, R. Lytel, and M. G. Kuzyk. Dalgarno-lewis perturbation theory for nonlinear optics. J. of the Opt. Soc. of Am. B, 33(12):E31, September 2016.

[46] A. A. Kocherzhenko, S. V. Shedge, X. S. Vazquez, J. Maat, J. Wilmer, A. F. Tillack, L. E. Johnson, and C. M. Isborn. Unraveling excitonic effects for the first hyperpolarizabilities of chromophore aggregates. J. of Phys. Chem. C, 123(22):13818-13836, February 2019.

[47] E. N. Svendsen. A variational method for calculation of dynamic third-order susceptibilities. Int. J. of Qua. Chem., 34(S22):477-480, 1988.

[48] J.-R. Gabryl, C. Barbier, P. Lemaire, and E. N. Svendsen. Variational calculation of the dynamic third-order susceptibility of water. J. of Mol. Str. Theochem, 357(3):199-205, 1995.

[49] J. C. G. Henriques, Høgni C. Kamban, Thomas G. Pedersen, and N. M. R. Peres. Calculation of the nonlinear response functions of intraexciton transitions in twodimensional transition metal dichalcogenides. Phys. Rev. B, 103:235412, Jun 2021.

[50] B. R. Johnson. New numerical methods applied to solving the one-dimensional eigenvalue problem. J. of Chem. Phys., 67(9):4086-4093, 1977.

[51] B. R. Johnson. The renormalized Numerov method applied to calculating bound states of the coupled-channel Schrödinger equation. J. of Chem. Phys., 69(10):46784688, 1978.

[52] G. V. Berghe, V. Fack, and H. E. de Meyer. Numerical methods for solving radial Schrödinger equations. J. of Comp. and Ap. Math., 28:391-401, 1989.

[53] J. C. G. Henriques, M. F. C. Martins Quintela, and
N. M. R. Peres. Theoretical model of the polarizability due to transitions between exciton states in transition metal dichalcogenides: application to wse2. J. Opt. Soc. Am. B, 38(7):2065-2074, Jul 2021.

[54] H. C. Kamban and T. G. Pedersen. Interlayer excitons in van der waals heterostructures: Binding energy, stark shift, and field-induced dissociation. Scientific Reports, 10(1), March 2020.

[55] T. G. Pedersen. Exciton stark shift and electroabsorption in monolayer transition-metal dichalcogenides. Phys. Rev. B, 94:125424, Sep 2016.

[56] A. Edery and P. Laporte. First and second-order relativistic corrections to the two and higher-dimensional isotropic harmonic oscillator obeying the spinless salpeter equation. J. Phys. Comm., 2(2):025024, feb 2018.

[57] B. Cohen-Tannoudji, F. Laloe, and D. Claude. Quantum Mechanics: volume 3. Wiley, 122019.

[58] M. Quintela and N. Peres. A colloquium on the variational method applied to excitons in $2 \mathrm{~d}$ materials. Eur. Phys. J. B, 93(12):222, 2020.

[59] Federico Grasselli. Variational approach to the softCoulomb potential in low-dimensional quantum systems. Am. J. Phys., 85(11):834-839, November 2017.

[60] E. Castaño. The confined two-dimensional hydrogen atom in the linear variational approach. Rev. Mex. de Fis., 51:6, 012005.

[61] H. E. Montgomery and T. G. Rubenstein. One-electron wavefunctions. Dynamic dipole polarizabilities. Chem. Phys. Let., 58(2):295-297, 1978.

[62] R. Yaris. Time-Dependent Perturbation-Variation Method. J. of Chem. Phys., 39(10):2474-2477, 1963.

[63] R. Yaris. Time-Dependent Perturbation-Variation Method. II. J. of Chem. Phys., 40(3):667-668, 1964.

[64] C. Robert, M. A. Semina, F. Cadiz, M. Manca, E. Courtade, T. Taniguchi, K. Watanabe, H. Cai, S. Tongay, B. Lassagne, P. Renucci, T. Amand, X. Marie, M. M. Glazov, and B. Urbaszek. Optical spectroscopy of excited exciton states in MoS2 monolayers in van der waals heterostructures. Phys. Rev. Mat., 2(1), January 2018.

[65] W. Kim, J. Y. Ahn, J. Oh, J. H. Shim, and S. Ryu. Second-harmonic young's interference in atom-thin heterocrystals. Nano Letters, 20(12):8825-8831, 2020.

[66] R. Boyd. Nonlinear Optics. Academic Press, London, 2020.

[67] A. Jeffrey and D. Zwillinger. Table of Integrals, Series, and Products. Academic Press, Amsterdam ; Boston, 8th edition edition, 2014. 